\title{
Adhesion and Anisotropic Friction Enhancements of Angled Heterogeneous Micro-Fiber Arrays with Spherical and Spatula Tips
}

July 30,2007

Michael P. Murphy, Burak Aksak, and Metin Sitti*

Mechanical Engineering Department, Carnegie Mellon University

Pittsburgh, PA 15213-3890, USA

*Corresponding author

Tel: 412-268-3632

Fax: 412-268-3348

E-Mail: msitti@andrew.cmu.edu

Short Title: Tip Enhancements of Angled Micro-Fiber Arrays 


\begin{abstract}
Angled polyurethane fiber arrays are modified by adding soft spherical and spatula shaped tips via dipping. These fibers are characterized for adhesion and friction and compared with unmodified fibers and flat material samples. Sphere and spatula tip fiber samples demonstrate increased adhesion, with 10 and 23 times the maximum adhesion of the unmodified fiber sample, respectively. The sphere and spatula tip fiber samples also show increased friction, with 1.6 and 4.7 times the maximum friction of the unmodified fiber sample, respectively. Friction and adhesion are simultaneously observed in a synthetic dry angled fibrillar adhesive sample (spatula tip fiber sample). The direction dependent friction of angled fibers is investigated. The adhesion and friction results reported in this paper suggest that fibers with negligible adhesion can be modified to exhibit both significant adhesion and friction enhancements by the proposed fiber tip modifications.
\end{abstract}

\title{
Keywords:
}

Gecko, Adhesion, Angled Fiber Array, Friction, Dry Adhesive, Bioinspired Adhesive 


\section{INTRODUCTION}

Biological dry adhesive pads found in geckos, spiders, and insects consist of arrays of micro and/or nano-scale hairs or fibers, often angled and with branching hierarchical structures [1]. The tips of these fibers have a spatula shape to increase the contact area with the adhering surface. These fibers bend and conform to the surface roughness of climbing surfaces, creating large contact areas from millions or billions of contact points. It has been shown that van der Waals forces $[2,3]$, possibly in combination with capillary forces $[4,5]$, are responsible for the resulting adhesion. Using these adhesive pads, geckos and other animals can efficiently climb on both smooth and rough surfaces with repeatable, controllable adhesion without degradation or contamination [6] for several months. It is essential that the adhesive pads provide both friction and adhesion simultaneously to facilitate climbing. For example, when climbing a vertical wall, a gecko's front feet provide adhesion forces to hold the animal to the wall, and at the same time provide shear forces that support the gecko's weight [7].

Since the discovery of the role of van der Waals forces in biological dry adhesives such as those in insects and geckos in 2002 [2], there has been a surge of interest in developing techniques for fabricating synthetic biomimetic dry adhesive materials for various applications, including climbing robots [8-11], and medical/surgical applications [12]. Researchers are characterizing biological samples $[3,13,14]$ and are fabricating synthetic adhesive arrays from polymers [15,16], carbon nanotubes [17,18], and organorods [19], using methods such as micro/nano-molding [16,20-25], nano-embossing [26], carbon nanotube growth [17,18], fiber drawing [27], and lithography $[15,28]$. Cylindrical polymer micro-pillars exhibit some of the characteristics of the biological dry adhesives, but have not shown a significant macro-scale adhesion performance increase over flat samples of the materials they are made from. Majidi et al. [20] have demonstrated significant friction enhancement with polypropylene fibers, but with negligible adhesion. Carbon nanotube arrays have also been tested which show high friction $[17,18]$. Recently, there has been success in fabricating vertical microscale fibers with spatula tip shapes similar to those seen in biological samples $[24,25,29,30]$. The spatula tipped fibers demonstrate significant adhesion performance over the cylindrical pillars as well as flat unstructured samples of the material from which they are made. Bhushan and Sayer [31] also demonstrated friction enhancement with spatula tipped fibers, however no adhesion data were presented.

In previous work [28], we have demonstrated the fabrication of angled microfibers and characterized their adhesive behavior. From analytical study and experiments it was determined that angled fibers, though more compliant, exhibited reduced adhesion compared to similar vertical fibers due to a peeling moment. However, from biological studies it is observed that angled fibers have been favored by evolution in many 
cases. Therefore, it stands to reason that the reduction in adhesion caused by the peeling moment can be overcome. We hypothesize that wider spatula tips may alleviate the peeling moment effect seen in angled fibers and lead to improved adhesive performance. To investigate this hypothesis, in this work, we modify angled polymer microfiber arrays by adding spherical and spatula tips to characterize the samples for adhesion and friction. In addition, the directional dependence of friction of synthetic angled fiber arrays is investigated.

In this paper, Section 2 describes a technique for modifying vertical or angled fibers by adding spherical or flat spatula tips. Experiments performed to characterize the macro-scale adhesion and friction of the fiber arrays are described in Section 3. The experimental results and observations are presented in Section 4. The results are compared with theory and discussed in Section 5. Finally, conclusions and future directions are reported in Section 6.

\section{Fiber Tip Fabrication}

In previous work we have described a technique for fabricating arrays of angled polyurethane microfibers [28], which were used as templates for further tip modification described in this section. A technique similar to the one developed by Arzt and coworkers [32,33] was used to modify these fibers by adding tip material in order to study the effect of specific tip shapes and softer tip material on adhesion and friction. The tip modification process is described in detail below. A lithographically formed master template of angled SU-8 fibers was first used to form a compliant negative mold. This mold was then used to form many copies of the fiber arrays from various curable polyurethanes by micro-molding. Since all polyurethane fiber arrays were fabricated from the same mold, they exhibited identical geometry and, therefore, provided an excellent starting point to examine tip effects on adhesion and friction performance. The backing layer for the fiber samples was approximately $2 \mathrm{~mm}$ thick.

To add tips to the template fibers, liquid polyurethane (ST-1060; BJB Enterprises, Tustin, CA) was mixed and spun onto a silicon wafer at $6000 \mathrm{rpm}$ for 5 minutes to form a thin uniform layer. This thin layer prevents excess polymer from seeping into the fiber samples during the dipping process. The wafer was then left for 30-40 minutes to increase the viscosity of the liquid polymer by partial curing. A fiber array sample was attached to a two-axis micro-positioning stage (UMR3.5; Newport, Irvine, CA) within the view of a top-view reflection type optical microscope (Eclipse L200; Nikon, Melville, NY). The wafer was attached to a two-axis goniometer (GON40-U; Newport, Irvine, CA) for alignment which was secured to a three-axis micro-positioning stage (M-461; Newport, Irvine, CA) across from the fiber array sample (Fig. 1a). The sample and wafer were aligned visually using the microscope on one edge, and by the eye on the other. After 
aligning the fiber sample and liquid layer, the sample was moved into contact with the liquid layer on the wafer and then retracted, retaining some of the liquid polymer on the tips of the fibers (Fig. 1b). To form spherical tips, the sample was placed with the fibers facing up and allowed to cure for 24 hours at room temperature. If spatula tips are desired, the fiber sample is instead placed onto a smooth low surface energy substrate immediately after dipping with the fibers facing down (Fig. 1c), and then peeled away once cured (Fig. 1d).
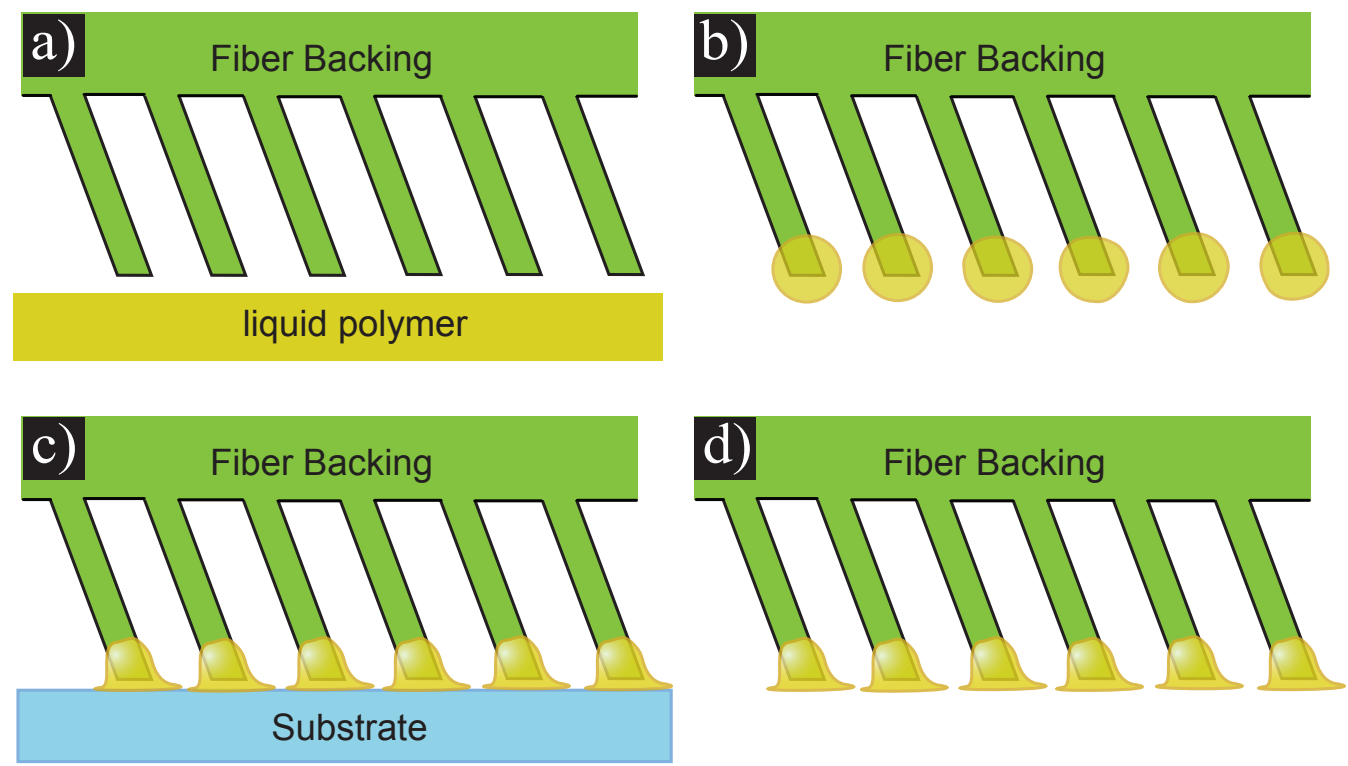

Figure 1: Fiber tip fabrication process: a) Bare fibers are aligned with a layer of liquid polymer; b) The fiber tips are dipped into the liquid and retracted; c) The fibers are brought into contact with a substrate; d) The fibers are peeled away from the substrate after curing.

One issue that can occur during the dipping process is incomplete coverage due to misalignment between the fiber sample and wafer, particularly in the direction not visible in the microscope view. Also, for longer, closely spaced, or more compliant fibers, the dipping process can result in fibers clumping together from capillary forces. If the liquid layer is too thin, or the polymer has low viscosity, the fibers do not collect enough tip material to form tip structures. Furthermore, if the liquid polymer wets the entire fiber due to low viscosity or material compatibility and does not remain concentrated at the tip, the tip formation process will not be successful.

When the fiber sample is placed on a substrate to form the spatula tips, consistent contact between the fibers and substrate is essential for tip shape uniformity. Fibers that are pressed completely form the desired spatula tips. If a fiber makes no contact with the substrate, a spherical cap is formed. If the liquid polymer 

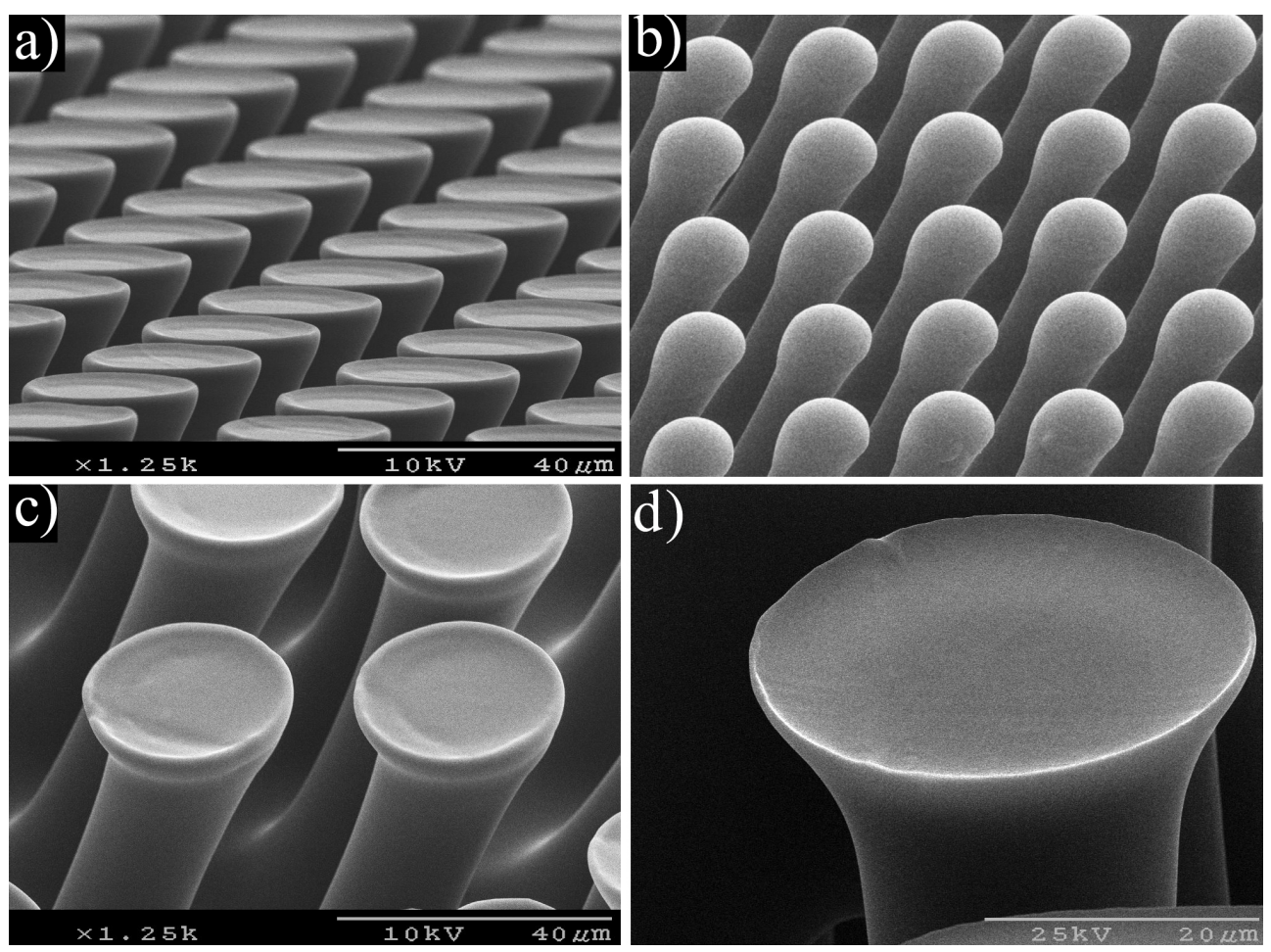

Figure 2: SEM micrographs of polymer fibers with: a) uniform spatula tips, b) uniform sphere tips, c) suction-cup-like spatula tips, and d) large diameter spatula tip ( $>1.6$ times base fiber diameter).

on the fiber touches the surface but there is a gap between the fiber and substrate, a liquid bridge is formed and results in a smaller tip shape. If the fibers have collected excess liquid polymer, the liquid drops merge to form fiber clumps. It is important to choose a substrate material which is smooth and has low surface energy, otherwise it was observed that the interface between the fiber and the tip broke when the sample was peeled off. A Fluoroware ${ }^{\circledR}$ mask holder was used as a substrate for successfully forming spatula tipped fibers.

This fabrication process yields spatula tips with varying sizes and shapes, depending on parameters such as retained liquid volume, material compatibility, and the force with which the fibers were pressed against the substrate. In cases with proper liquid volume and uniform loading, excellent tip uniformity is achieved (Fig. 2a). Consistent liquid volume and compatible materials yield uniform arrays of sphere tipped fibers (Fig. 2b). In many cases, the spatula tips form into suction-cup-like structures (Figs. 2a,c). This is possibly due to plastic deformation which occurs when peeling the fibers from the substrate. Fiber tips have been fabricated whose diameter is in excess of $160 \%$ of the base fiber diameter (Fig. $2 \mathrm{~d}$ ). 

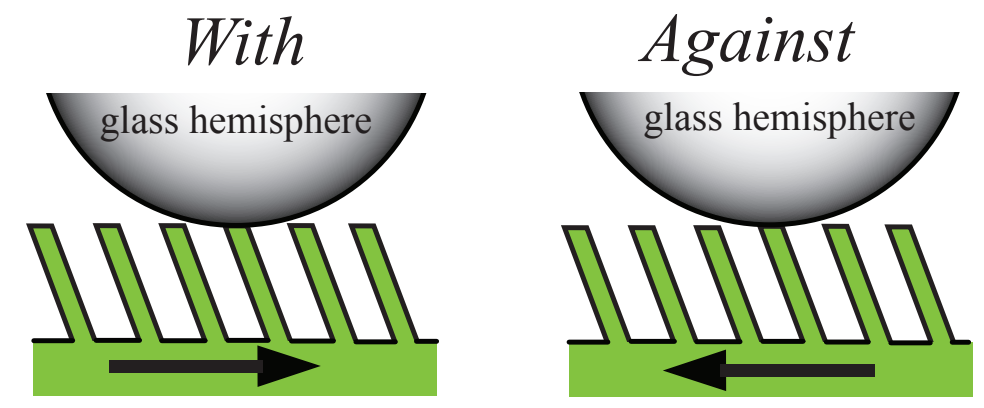

Figure 3: Illustration of the with and against directions of motion.

\section{Experimental}

\subsection{Materials and Methods}

A custom macroscale adhesion measurement system consists of an inverted optical microscope (Eclipse TE200; Nikon, Melville, NY) with an automated high precision stage (MFA-CC; Newport, Irvine, CA) which holds a high resolution load cell (GSO-25; Transducer Techniques Inc., Temecula, CA). A 6 mm diameter glass hemisphere (QU-HS-6; ISP Optics, Irvington, NY) is connected to the load cell. The fiber array samples are placed on the microscope stage with the fibers facing up toward the glass hemisphere. Custom real-time software controls the stage to lower the hemisphere into contact with the fiber sample at a fixed velocity until a pre-specified preload $\left(P_{p}\right)$ is reached. The hemisphere is then retracted at a speed of $2 \mu \mathrm{m} / \mathrm{s}$ until it detaches from the sample. The maximum separation force, or adhesion, $\left(P_{a}\right)$ is recorded during retraction. Slow preload and retraction speeds are preferred in order to reduce viscoelastic effects. However, sensor drift was observed in experiments at speeds slower than $2 \mu \mathrm{m} / \mathrm{s}$ due to the increased time-scale; therefore, $2 \mu \mathrm{m} / \mathrm{s}$ speed was chosen to minimize both viscoelastic effects and sensor drift. By varying the preload from 1 to $16 \mathrm{mN}$ and conducting many adhesion experiments, we created a performance curve for each sample by plotting the maximum adhesion $\left(P_{a}\right)$ vs. the preload $\left(P_{p}\right)$.

Friction characterization experiments were performed on a modified setup where the sample is affixed to a glass slide connected to a second load cell (GSO-50; Transducer Techniques Inc., Temecula, CA) to record the friction forces in the lateral direction. The hemisphere is brought into contact with the sample at $5 \mu \mathrm{m} / \mathrm{s}$ until preload is reached. After pausing for 1 second, the fiber sample is then moved in the lateral direction at a fixed velocity $(5 \mu \mathrm{m} / \mathrm{s})$ for a distance of $300 \mu \mathrm{m}$, followed by the retraction of the hemisphere from the sample until detachment occurs. The software continually captures the normal force and the friction force data from the load cells with $0.1 \mathrm{mN}$ force resolution. Although a target preload is set, the load conditions during the friction measurements are displacement driven, meaning it is the indentation depth, not the preload, that is 
kept constant during the lateral movement. The stiffness of the load cell stem $(2400 \mathrm{~N} / \mathrm{m})$ is high compared to the fiber array $(120 \mathrm{~N} / \mathrm{m})$, and thus it can be assumed rigid.

A hemispherical indenter was used rather than a flat-punch indenter for both adhesion and friction measurements to eliminate misalignment. Another advantage of this setup is that a hemispherical surface represents a special case of a rough surface with a well-defined height distribution.

To investigate the anisotropy of friction for the angled fiber arrays, friction measurements were performed in two directions, with the angle and against the angle (Fig. 3). Friction in the direction perpendicular to the fiber angle was not characterized.

A profile view system [28] was used to observe the qualitative behavior of the fibers interacting with the test glass hemisphere during loading, unloading, and lateral displacement conditions. The observations are discussed in Section 4.

\subsection{Samples}

Table 1: Material Specifications.

\begin{tabular}{|c|c|c|c|}
\hline Material & Young's Modulus $(E)$ & Poisson's Ratio $(\nu)$ & Work of Adhesion $\left(w_{f}\right)$ \\
\hline ST-1087 & $9.8 \mathrm{MPa}$ & 0.5 & $32 \mathrm{~mJ} / \mathrm{m}^{2}$ \\
ST-1060 & $2.9 \mathrm{MPa}$ & 0.5 & $93 \mathrm{~mJ} / \mathrm{m}^{2}$ \\
\hline
\end{tabular}

Five sample types in $1.5 \mathrm{~mm}$ by $1.5 \mathrm{~mm}$ arrays were prepared for characterization from two different polyurethanes (ST-1087, ST-1060; BJB Enterprises, Tustin, CA). The material properties of these polyurethanes are detailed in Table 1. The Young's moduli were determined by tensile testing of bulk samples, and the effective work of adhesion was estimated by Atomic Force Microscopy experiments using a $12 \mu \mathrm{m}$ diameter borosilicate particle probe, and the Poisson's ratio was assumed to be 0.5 as is common with elastomer polymers. All of the fiber array templates were fabricated as described in previous work [28] and had identical fiber geometry; the fiber diameter was $19 \mu \mathrm{m}$, the length was $92 \mu \mathrm{m}$, and the angle with respect to vertical was $20^{\circ}$. The fibers were arranged in a square grid pattern with $40 \mu \mathrm{m}$ center-to-center spacing. Fiber array samples without additional tip structures (Fig. 4a) were prepared from both materials as control samples, and are referred to as unmodified samples. Also, ST-1087 fibers were modified to have spherical (Fig. 4b) or spatula (Fig. 4c) tips made from the softer ST-1060 polyurethane. The fiber and tip materials for each sample are listed in Table 2 along with the tip geometry parameters. In addition, a flat unstructured sample of each of the materials was prepared for comparison. 

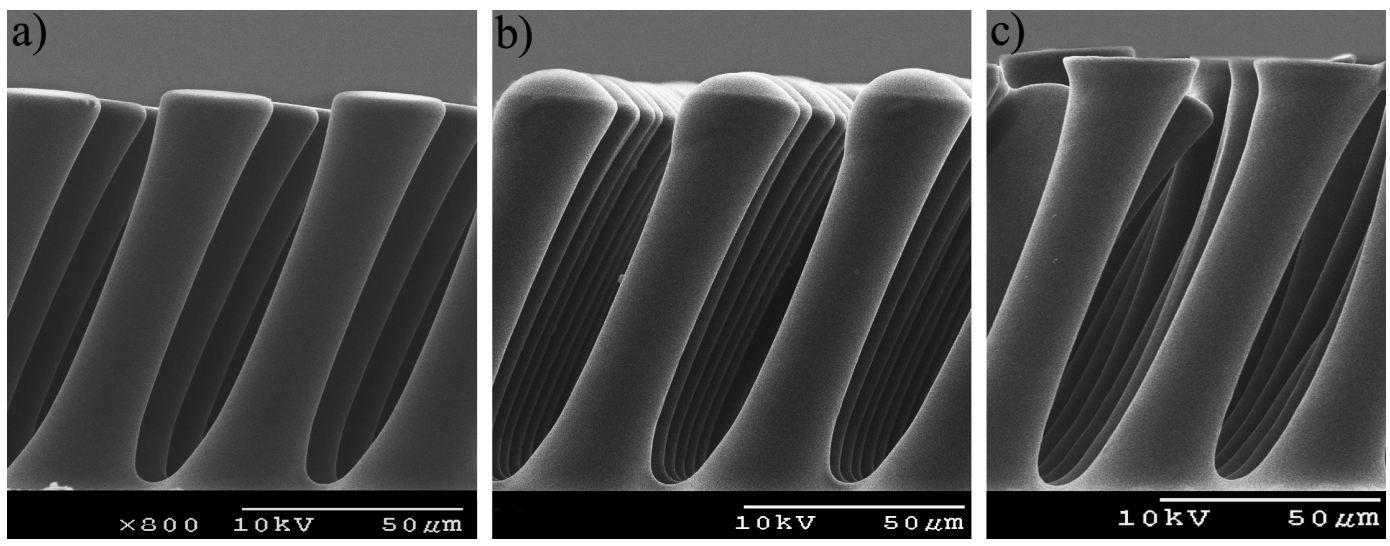

Figure 4: SEM images of polyurethane fibers used in experiments with: a) unmodified flat tips, b) spherical tips, and c) spatula tips.

Table 2: Sample Specifications.

\begin{tabular}{|c|c|c|c|c|}
\hline & Spatula Tip & Sphere Tip & Unmodified ST-1087 & Unmodified ST-1060 \\
\hline Fiber Material & ST-1087 & ST-1087 & ST-1087 & ST-1060 \\
\hline Tip Material & ST-1060 & ST-1060 & ST-1087 & ST-1060 \\
\hline Tip Shape & Spatula & Spherical & Flat Punch & Flat Punch \\
\hline Tip Radius & $16 \pm 1 \mu \mathrm{m}$ & $17 \mu \mathrm{m}^{*}$ & $9.5 \mu \mathrm{m}$ & $9.5 \mu \mathrm{m}$ \\
\hline Total Length & $103 \mu \mathrm{m}$ & $95 \mu \mathrm{m}$ & $92 \mu \mathrm{m}$ & $92 \mu \mathrm{m}$ \\
\hline Effective Young's Modulus $^{\dagger}$ & $200 \mathrm{kPa}$ & $200 \mathrm{kPa}$ & $200 \mathrm{kPa}$ & $160 \mathrm{kPa}$ \\
\hline
\end{tabular}

* Radius of curvature

$\dagger$ Estimated from fitting experimental data to Hertzian indentation theory [30]

\section{Results}

\subsection{Adhesion}

Fifteen adhesion experiments were performed on each sample detailed in Table 2 with preloads from $1 \mathrm{mN}$ to $15 \mathrm{mN}$. The adhesion results are plotted together in Fig. 5 to compare their relative adhesion performances. The unmodified ST-1087 fibers exhibit up to $1 \mathrm{mN}$ of adhesion at low preloads, and no adhesion within the sensitivity of the load cell for higher preloads. The fibers fabricated from the softer ST-1060 polyurethane (unmodified ST-1060) demonstrated higher adhesion at all preloads saturating at $2.8 \mathrm{mN}$. The sphere tipped fibers exhibit similar saturation behavior to the unmodified ST-1060 fibers, but a higher overall and saturation adhesion $(6 \mathrm{mN})$. The fiber arrays with spatula tips demonstrated significantly higher adhesion than all of the other fiber array samples. The adhesion performance increases with preload and the trend of the data does not indicate a saturation in tested preload range. The maximum recorded adhesion from the spatula tipped 
fiber array was 23 times the maximum adhesion of the unmodified ST-1087 fibers, 5 times the maximum adhesion of the unmodified ST-1060 fibers, and 2.3 times the maximum adhesion of the sphere tipped fibers.

To determine if the spatula tipped fiber array sample demonstrated adhesion properties superior to the flat unstructured polyurethane materials, they were also tested and the results were plotted in Fig. 5. The flat ST-1087 sample saturates at approximately $5 \mathrm{mN}$, whereas the softer flat ST-1060 sample exhibits a maximum adhesion of $12.5 \mathrm{mN}$. Similar to the spatula tipped fiber sample, the flat ST-1060 sample does not show saturation characteristic within the range of preloads in the tests. The maximum adhesion of the spatula fiber sample is 2.7 times the flat ST-1087 sample, and approximately 1.1 times the flat ST-1060 sample.

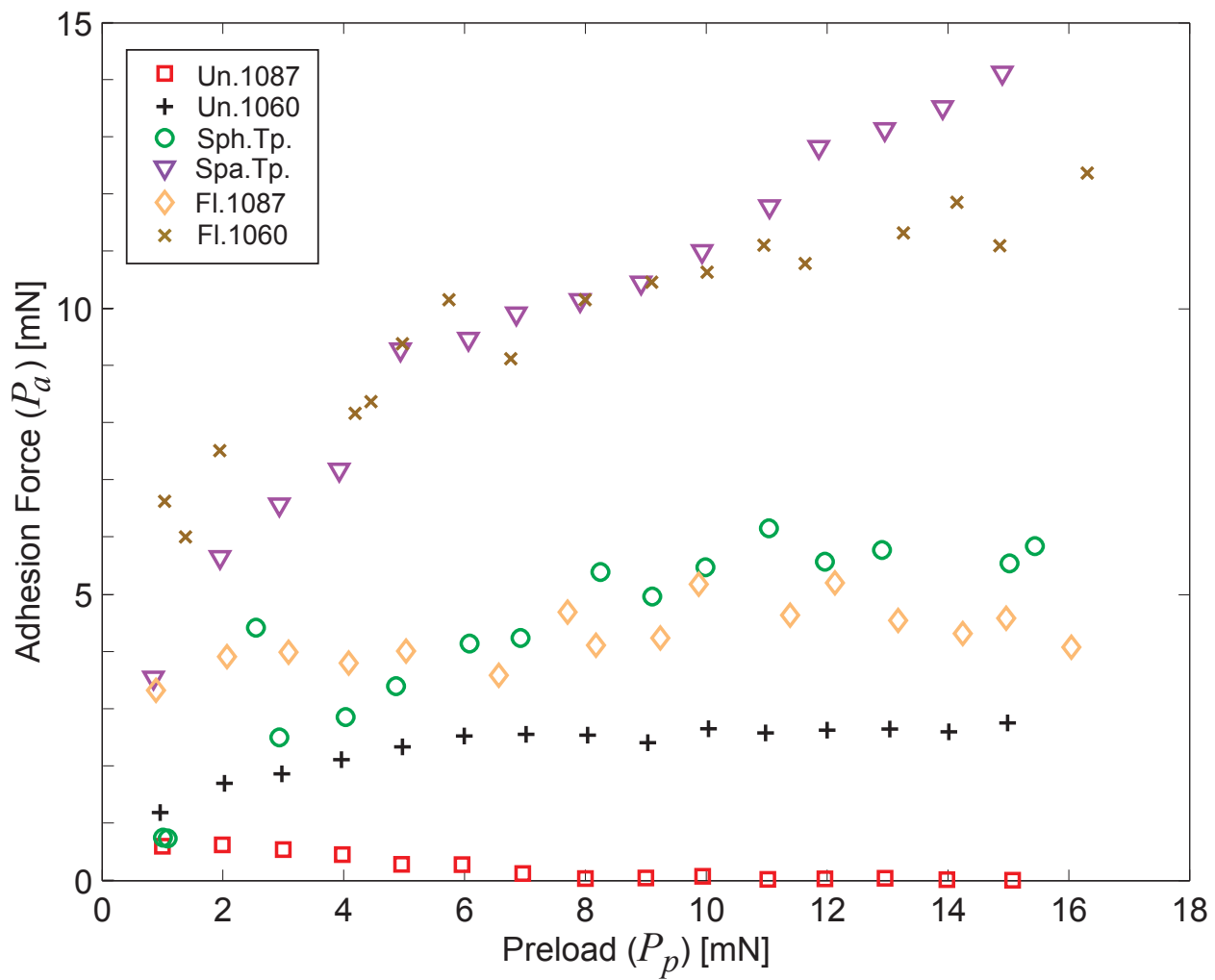

Figure 5: Adhesion force vs. preload data. The spatula tipped fiber sample exhibits the highest adhesion performance, 23 times the maximum adhesion of the unmodified ST-1087 fibers.

Visual observations indicate that the unmodified ST-1087 fibers exhibit tip contact loss under compressive loading and very little extension when the sphere is retracted (Fig. 6a). The spherical tips were observed to eliminate tip contact loss under compressive loading, and although the maximum fiber extension is small, it was observed that multiple fibers extend together before pull-off occurs (Fig. 6b). The unmodified ST-1060 fibers buckled under high compressive loading, but maintained full tip contact and exhibited high extension 
prior to pull-off (Fig. 6c). Similarly, the spatula tipped fibers maintained tip contact under compressive loading and demonstrated high extension under tension (Fig. 6d).

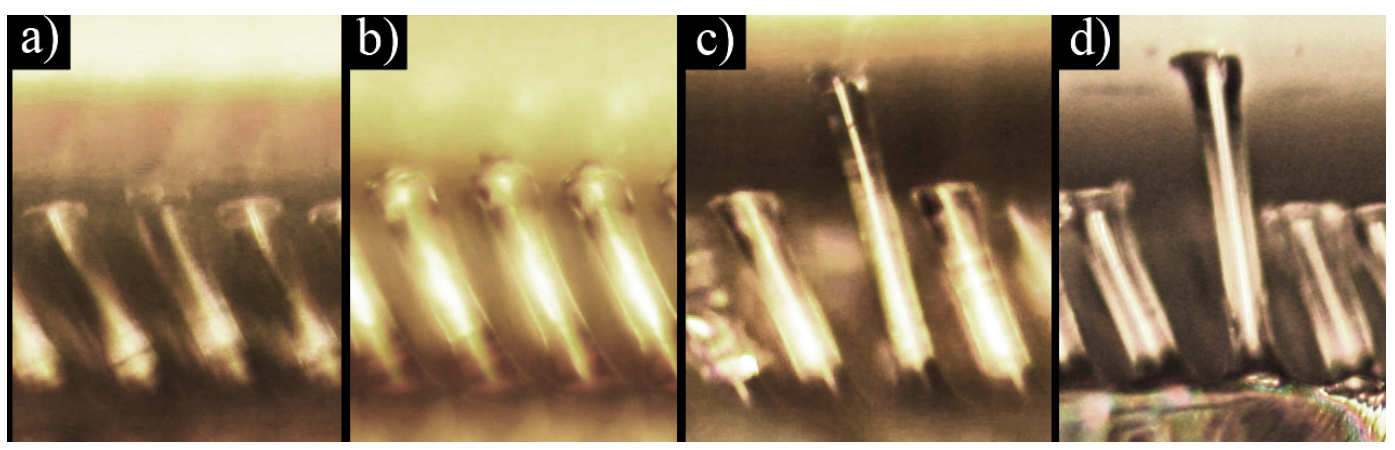

Figure 6: Profile view images of fiber array samples just before pull-off occurs for a) unmodified ST-1087 fibers, b) sphere tip fibers, c) ST-1060 unmodified fibers, and d) spatula tip fibers.

\section{$4.2 \quad$ Friction}

Friction characterization was performed on the unmodified ST-1087 and ST-1060, flat ST-1060, sphere tip, and spatula tip samples. Each sample was tested at $2 \mathrm{mN}$ increments between $1 \mathrm{mN}$ and $16 \mathrm{mN}$ preloads in both the with (Fig. 7a-d) and against (Fig. 7e-h) directions. Force vs. time data from the $4 \mathrm{mN}$ preload experiments are presented in Fig. 7. The dotted lines represent normal forces (compression/adhesion) and the solid lines represent lateral forces (friction). In each plot, the preload is represented by the initial rise in normal force data. At this point, indicated by a vertical line, the sample begins to move laterally and the data recorded represent the friction and normal forces generated during dragging. At the second vertical line the sphere is retracted.

The normal force data from the with direction (Fig. 7a-d) decrease immediately when the lateral motion is initiated due to fiber extension. In contrast, in the against direction experiments (Fig. 7e-h), the normal force initially rises before eventually decreasing due to fiber compression. This trend was observed at all preloads for all fiber samples.

The unmodified ST-1087, unmodified ST-1060, and sphere tip samples exhibit friction in both the with and against directions, but exhibit no significant adhesion during dragging. However, the spatula tip array sample demonstrates adhesion and friction simultaneously in both directions (Fig. 7d,h). Furthermore, the friction is significantly enhanced compared to the other three samples, particularly comparing the data from the with direction. Upon retraction, the spatula tip sample exhibits a sharp rise in adhesion.

The results from the friction tests are plotted together in Fig. 8. The preload values in the plot correspond 


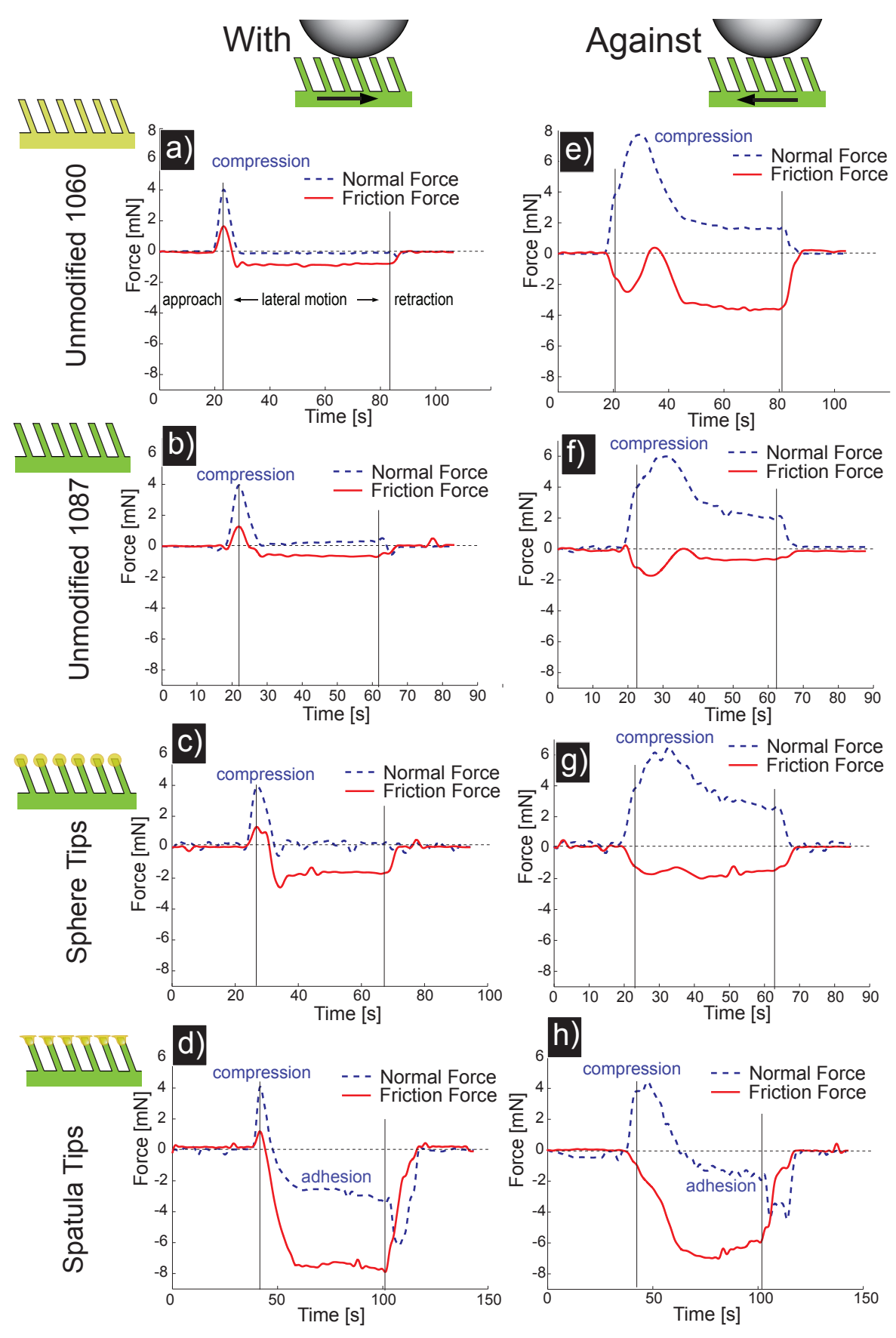

Figure 7: Normal and friction force data vs. time for $4 \mathrm{mN}$ preload for the four fiber sample types (unmodified ST-1060, unmodified ST-1087, sphere tips, spatula tips). Rows represent sample type, columns represent the direction of relative lateral motion. Vertical and lateral speeds were $5 \mu \mathrm{m} / \mathrm{s}$.

to the applied compressive load prior to lateral motion, and the friction force is the maximum lateral force (negative peak in Fig. 7) recorded against the direction of motion. In all of the tests, the flat sample demonstrated higher friction than any of the fiber samples. Of the fibrillar samples, the highest friction 
forces are observed in the spatula tip fibers in the with direction. The against direction friction values for these fibers are greater at low preloads, but slightly lower at higher preloads. The sphere tip fibers and the spatula tip fibers demonstrated $56 \%$ and $37 \%$ higher friction in the with direction, respectively. In contrast, the unmodified ST-1087 and unmodified ST-1060 samples exhibit 60\% and 109\% higher friction in the against direction, respectively, with the unmodified ST-1087 sample exhibiting overall lowest friction among the samples tested. The maximum friction recorded for the spatula fibers is 2.8 times the maximum sphere tip friction, and 4.7 and 1.66 times the highest recorded friction for the unmodified ST-1087 and ST-1060 fiber samples, respectively.

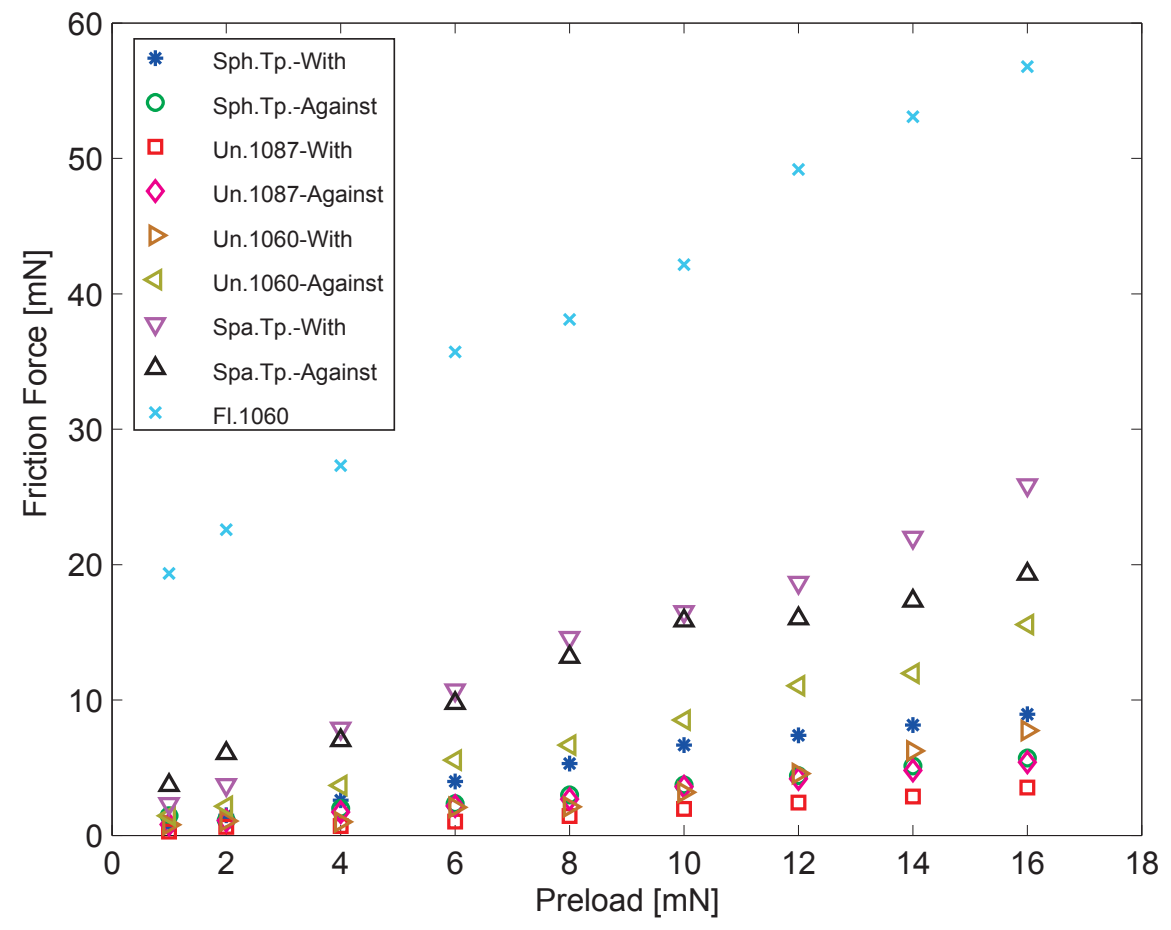

Figure 8: Maximum friction force vs. applied preload from the friction experiments for the spatula tip fiber sample (Spa.Tp.), sphere tip fiber sample (Sph.Tp.), unmodified ST-1060 sample (Un.1060), unmodified ST-1087 sample (Un.1087), and flat control ST-1060 sample (Fl.1060).

Observing the fiber behavior under lateral loading in the microscope profile view, it is clear that the unmodified ST-1087 tip surfaces of the fibers lose contact with the glass sphere in the with direction immediately upon lateral motion (Fig. 9a). In the against direction, the fibers are flipped backward and dragged, again with only the edge of the fiber tip in contact (Fig. 9d). Although the contact points on the tips change, the sphere tip fibers are observed to maintain tip contact when dragged in both directions (Fig. 9b,e) due to the symmetry of their spherical tip shape. The spatula tip fibers are observed to maintain full tip contact 
for larger lateral displacements before transitioning to edge contact (Fig. 9c,f) in both the with and against directions.

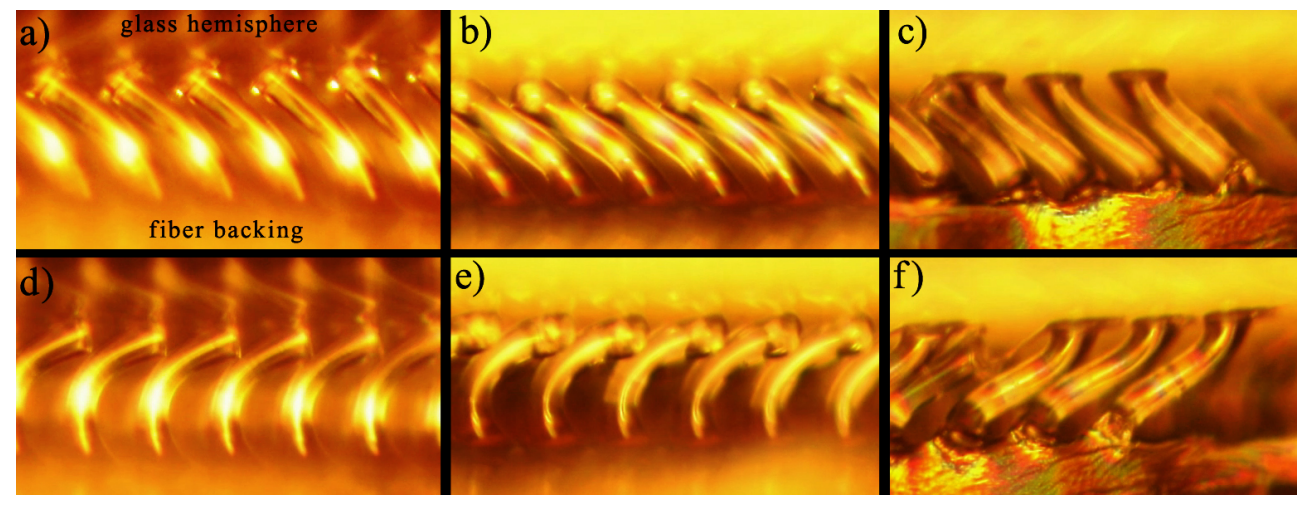

Figure 9: Optical microscope side view of fiber samples during lateral motion in the with direction (a,b,c) and against direction (d,e,f) for the unmodified ST-1087 sample (a,d), sphere tip sample (b,e), and spatula tip sample (c,f). The unmodified ST-1087 fibers have only edge contact, the sphere tip fibers have sphere contact points, and the spatula tips maintain full tip contact for relatively large lateral tip displacements.

\section{Discussion}

\subsection{Adhesion}

From a beam mechanics perspective, the main advantage of angled fibers, with respect to adhesion, is enhanced compliance. Angled fibers experience bending in addition to axial compression/extension during loading/unloading which results in high deflection. From this, it is expected that angled fibers generate high overall adhesion. However, it is shown that the adhesion of a single angled fiber is reduced due to the additional asymmetric stress created by the moment at the tip of the fiber which changes the detachment mode from uniform pull-off to peeling. Without reducing the stress caused by the moment, overall adhesion enhancement of angled fibers has not been demonstrated. A detailed analysis and the experimental results can be found in [28].

The average tensile stress component $\left(\sigma_{M}\right)$ caused by the moment at the tip is approximated by

$$
\sigma_{M}=\frac{16 M}{3 \pi^{2} r_{t}^{3}}
$$

where $r_{t}$ is the tip radius, and $M$ is the moment caused by the fiber deflection which is constrained by a guided-fixed boundary condition as described in [28]. Since this average stress has an inverse cubic dependence on tip radius, increasing the tip radius should reduce the stress drastically. The spatula tip samples have a 
tip radius which is up to 1.78 times the unmodified fibers. According to Eq. (1), this reduces the average tensile stress component caused by the moment by $82 \%$.

A displacement based MATLAB ${ }^{\circledR}$ simulation, detailed in [28], which models the macroscale behavior of fiber arrays in contact with a spherical surface, was modified to allow for changes in tip radius. Simulations were performed for unmodified ST-1087, unmodified ST-1060, and spatula tip samples using the parameters from Tables 1 and 2, interacting with a $6 \mathrm{~mm}$ diameter glass sphere. The simulation results are plotted along with the experimental data for these three samples in Fig. 10. In general, the experimental results match closely with the theoretical predictions, indicating that the analytical model is effective in predicting adhesion performance. Theory assumes full tip contact at all preloads and, therefore, does not predict the reduced adhesion due to tip contact loss at high preloads for the unmodified ST-1087 fibers. The wide region for simulated spatula tip sample adhesion illustrates the high sensitivity of adhesion to fiber tip radius.

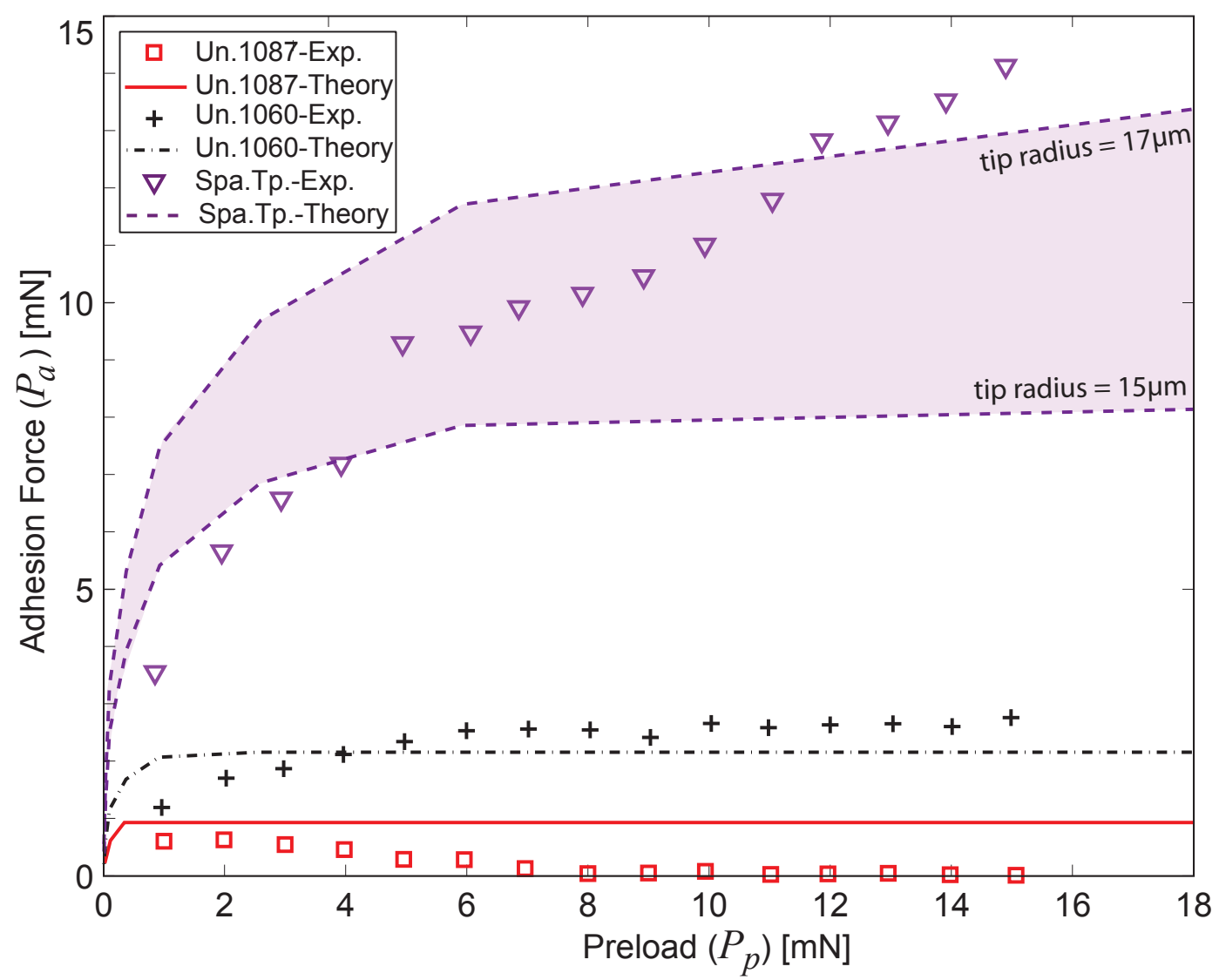

Figure 10: Experimental adhesion force vs. preload results compared with theoretical predictions.

The sphere tip fibers have unfavorable tip geometry due to the reduced contact area compared to unmodified tips. Despite this disadvantage, the sphere tip sample exhibits higher adhesion than the unmodified 
ST-1087 fibers in our experiments. This enhancement can be attributed to the softer, higher surface energy tip material. Therefore, the adhesion performance enhancement of the spatula tip fibers cannot be explained by tip size alone - the material properties of the spatula tip are also favorable for high adhesion. Also, SEM images (Fig. 2a,c,d) suggest that the spatula tips may act as micro suction cups. If this is the case, there may be an additional, unmodeled, contribution to the overall adhesion.

Profile view observations indicate that the maximum extension of the spatula tip fibers is the same as the maximum extension of the unmodified ST-1060 fibers. A linear spring model [28] for fiber deflection suggests that fiber stiffness is linearly dependent on Young's modulus. Therefore, the spatula tip fibers that are fabricated from ST-1087 are more than three times stiffer than the unmodified ST-1060 fibers. This means that, given the same extension, the single spatula tip fiber is providing at least 3 times as much adhesion. This estimation is consistent with the experimental data in Fig. 10, particularly for mid-range preloads.

\subsection{Friction}

The friction force measured in the experiments is a combination of two types of forces: friction that is governed by Coulomb's law, and "adhesive friction." Coulomb's friction occurs when a fiber is in compression whereas adhesive friction is the lateral component of the tensile force on the fiber. To determine the nature of the friction force in the experiments, the contact of the glass sphere with the fiber array should be studied in detail. Due to the profile of the glass sphere used in the experiments, each fiber is deflected a different amount. Some fibers may be in tension while others are in compression. Also, when the lateral motion begins, some fibers are already in contact and eventually pull off, whereas others come into contact as the sample moves. However, the normal force data only represent the sum of the normal forces on each fiber. Therefore, even when the normal force on the sphere is compressive, some of the friction force may be generated by the fibers in tension (adhesive friction), making it difficult to draw conclusions about the exact nature of the friction from the experimental data.

Despite the unclear nature of the friction, one conclusion can be reached: the spatula tip fiber arrays exhibit adhesion and friction simultaneously. This result is similar to the friction experiments conducted by Autumn et al. [13] with isolated tokay gecko setae on a flat glass surface, where the data also show adhesion and friction simultaneously in the with direction.

The directional dependence of the friction in the modified tip samples tested was evident, but not drastic. For example, even in the against direction, the spatula tip samples exhibit significantly higher friction than the sphere tip and unmodified ST-1087 samples in either direction. Early tip contact loss in the against 
direction would have caused a drastic reduction in the spatula tip sample friction, but was not observed (Fig. 9f). This could be due to the relatively small fiber angle. For larger fiber angles, the anisotropy may increase significantly if the lateral motion in the against direction causes earlier tip contact loss, while the with direction behavior does not change significantly.

\section{Conclusions}

Angled polyurethane fiber arrays were modified by adding soft tips in spherical and spatula shapes by dipping. These fibers were characterized for adhesion and friction and compared with the unmodified fibers and flat material samples. The sphere and spatula tip fiber samples demonstrate increased adhesion, with 10 times and 23 times the maximum adhesion of the unmodified ST-1087 sample, and 2.2 times and 5 times the the maximum adhesion of the unmodified ST-1060 sample, respectively. The spatula tip fiber sample also demonstrated higher adhesion than both flat samples. The macro-scale fiber array adhesion model from [28] was modified to allow for changes in tip radius, and the results were consistent with the experimental data.

The sphere and spatula tip fiber samples also show increased friction, with 1.6 and 4.7 times the maximum friction of the unmodified ST-1087 sample, respectively. The spatula tip fiber sample exhibited 1.66 times the maximum friction of the unmodified ST-1060 sample, while the unmodified ST-1060 sample demonstrated 1.75 times the maximum friction of the sphere tip sample. Simultaneous friction and adhesion were observed in the spatula tip fiber sample. The direction dependent friction of angled fibers was also investigated. The friction in the with direction was found to be up to $56 \%$ higher than the friction in the against direction for the samples with modified tips. The unmodified ST-1087 amd ST-1060 samples exhibited 60\% and 109\% higher friction in the against direction, respectively.

The adhesion enhancement observed for the spatula tip sample indicates that the stress caused by the moment at the tip of angled fibers can be alleviated by a larger tip radius. Also, the adhesion enhancement of the sphere tip fibers over the unmodified ST-1087 sample observed in the experiments suggests that adding compliant tips to stiff fibers improves adhesion and friction performance. The adhesion and friction results reported in this paper suggest that fibers with negligible adhesion can be modified to exhibit significant adhesion and friction enhancement by the proposed post-fabrication tip modifications. This finding opens the door to new fabrication techniques which can create fiber arrays from materials which are not highly adhesive, but can be easily modified to exhibit enhanced adhesion and friction by adding tips. Future work will include modeling the friction experiments and comparing angled fibers with spatula tips to vertical fibers with similar tips. 


\section{Acknowledgments}

The authors would like to acknowledge all of the members of the NanoRobotics Laboratory, especially Chytra

Pawashe, Eugene Cheung, and Seok Kim for building the friction measurement system. 


\section{References}

[1] E. Arzt, S. Gorb, and R. Spolenak, Proc. Nat1. Acad. Sci. USA 100, 10603-10606 (2003).

[2] K. Autumn, M. Sitti, Y. A. Liang, A. M. Peattie, W. R. Hansen, S. Sponberg, T. W. Kenny, R.

Fearing, J. N. Israelachvili, and R. J. Full, Proc. Natl. Acad. Sci. USA 99, 12252-12256 (2002).

[3] K. Autumn, Y. A. Liang, S. T. Hsieh, W. Zesch, W. P. Chan, T. W. Kenny, R. Fearing, and R. J. Full, Nature 405, 681-685 (2000).

[4] G. Huber, H. Mantz, R. Spolenak, K. Mecke, K. Jacobs, S. Gorb, and E. Arzt, Proc. Natl. Acad. Sci. USA 102, 16293-16296 (2005).

[5] W. Sun, P. Neuzil, T. Kustandi, S. Oh, and V. D. Samper, Biophysical J.: Biophysical Letters, L14L17 (2005).

[6] W. Hansen, and K. Autumn, Proceedings Proc. Natl. Acad. Sci. USA 102, 385-389 (2005).

[7] K. Autumn, S. T. Hsieh, D. M. Dudek, J. Chen, C. Chitaphan, and R. J. Full, J. Exprl. Biol. 209, 260$272(2006)$.

[8] M. P. Murphy, and M. Sitti, IEEE/ASME Transactions on Mechatronics 12, 330-338 (2007).

[9] O. Unver, A. Uneri, A. Aydemir, and M. Sitti, Proc. IEEE International Conference on Robotics and Automation, Volume 1, pp. 2329-2335, (2006).

[10] K., Daltorio, S. Gorb, A. Peressadko, A. Horchler, R. Ritzmann, and R. Quinn, Proc. International Conference on Climbing and Walking Robots, pp. 131-138, (2005).

[11] S. Kim, M. Spenko, S. Trujillo, B. Heyneman, V. Mattoli, and M. Cutkosky, Proc. IEEE International Conference on Robotics and Automation, pp. 1268-1273, (2007).

[12] J. Kwon, E. Cheung, S. Park, and M. Sitti, Biomedical Mater. 1, 216-220 (2006).

[13] K. Autumn, A. Dittmore, D. Santos, M. Spenko, and M. Cutkosky, J. Exprl. Biol. 209, 3569-3579 (2006).

[14] K. Autumn, C. Majidi, R. E. Groff, A. Dittmore, and R. Fearing, J. Exprl. Biol. 209, 3558-3568 (2006).

[15] A. K. Geim, S. V. Dubonos, I. V. Grigorieva, K. S. Novoselov, A. A. Zhukov, and S. Y. Shapoval, Nature Materials 2, 461-463 (2003).

[16] N. J. Glassmaker, A. Jagota, C.-Y. Hui, and J. Kim, J. Royal Soc. Interface 1, 23-33 (2004). 
[17] Y. Zhao, T. Tong, L. Delzeit, A. Kashani, M. Meyyappan, and A. Majumdar, J. Vacuum Sci. \& Technol. B 24, 331-335 (2006).

[18] P. Dickrell, S. Sinnott, D. Hahn, N. Raravikar, L. Schadler, P. Ajayan, and W. Sawyer, Tribology Letters 18, 59-62 (2005).

[19] M. T. Northen, and K. L. Turner, Nanotechnology 16, 1159-1166 (2005).

[20] C. Majidi, R. E. Groff, Y. Maeno, B. Schubert, S. Baek, B. Bush, R. Maboudian, N. Gravish, M. Wilkinson, K. Autumn, and R. S. Fearing, Phys. Rev. Letters 97, 076103 (2006).

[21] M. Sitti, and R. Fearing, J. Adhesion Sci. and Technol. 17, 1055-1074 (2003).

[22] C. Majidi, R. Groff, and R. Fearing, Proc. of the ASME International Mechanical Engineering Congress and Exposition, 579-584 (2004).

[23] C. Menon, M. Murphy, and M. Sitti, Proc. of the IEEE Int. Conf. on Robotics and Biomimetics, pp. 431-436 (2004).

[24] S. Kim, and M. Sitti, Appl. Phys. Letters 89, 261911 (2006).

[25] S. Gorb, M. Varenberg, A. Peressadko, and J. Tuma, J. Royal Soc. Interface 4, 271-275 (2007).

[26] D. S. Kim, H. S. Lee, J. Lee, S. Kim, K.-H. Lee, W. Moon, and T. H. Kwon, Microsystem Technologies 13, 601-606 (2007).

[27] G. L. Spina, C. Stefanini, A. Menciassi, and P. Dario, J. Micromech. Microeng. 15, 1576-1587 (2005).

[28] B. Aksak, M. Murphy, and M. Sitti, Langmuir 23, 3322-3332 (2007).

[29] A. Del Campo, and E. Arzt, Macromolecular Bioscience 7, 118-127 (2007).

[30] C. Greiner, A. Del Campo, and E. Arzt, Langmuir 23, 3495-3502 (2007).

[31] B. Bhushan, and R. A. Sayer, Microsystem Technologies 13, 71-78 (2006).

[32] A. D. Campo, C. Greiner, I. Alvarez, and E. Arzt, Adv. Mater. 19, 1973-1977 (2007).

[33] A. D. Campo, C. Greiner, and E. Arzt, Langmuir (2007). in review. 


\section{List of Figures}

1 Fiber tip fabrication process: a) Bare fibers are aligned with a layer of liquid polymer; b) The fiber tips are dipped into the liquid and retracted; c) The fibers are brought into contact with a substrate; d) The fibers are peeled away from the substrate after curing. . . . . . . . . . . 5

2 SEM micrographs of polymer fibers with: a) uniform spatula tips, b) uniform sphere tips, c) suction-cup-like spatula tips, and d) large diameter spatula tip ( $>1.6$ times base fiber diameter). 6

$3 \quad$ Illustration of the with and against directions of motion. . . . . . . . . . . . . 7

4 SEM images of polyurethane fibers used in experiments with: a) unmodified flat tips, b)

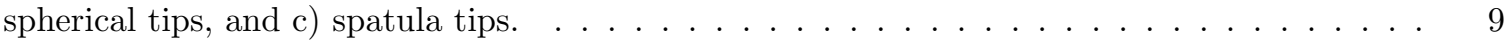

5 Adhesion force vs. preload data. The spatula tipped fiber sample exhibits the highest adhesion performance, 23 times the maximum adhesion of the unmodified ST-1087 fibers. . . . . . . 10

6 Profile view images of fiber array samples just before pull-off occurs for a) unmodified ST-1087 fibers, b) sphere tip fibers, c) ST-1060 unmodified fibers, and d) spatula tip fibers. . . . . . . 11

7 Normal and friction force data vs. time for $4 \mathrm{mN}$ preload for the four fiber sample types (unmodified ST-1060, unmodified ST-1087, sphere tips, spatula tips). Rows represent sample type, columns represent the direction of relative lateral motion. Vertical and lateral speeds

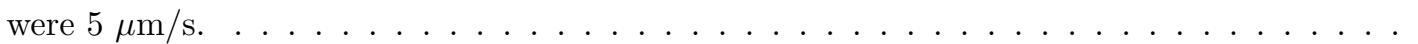

8 Maximum friction force vs. applied preload from the friction experiments for the spatula tip fiber sample (Spa.Tp.), sphere tip fiber sample (Sph.Tp.), unmodified ST-1060 sample (Un.1060), unmodified ST-1087 sample (Un.1087), and flat control ST-1060 sample (Fl.1060).

9 Optical microscope side view of fiber samples during lateral motion in the with direction $(\mathrm{a}, \mathrm{b}, \mathrm{c})$ and against direction (d,e,f) for the unmodified ST-1087 sample (a,d), sphere tip sample (b,e), and spatula tip sample $(\mathrm{c}, \mathrm{f})$. The unmodified ST-1087 fibers have only edge contact, the sphere tip fibers have sphere contact points, and the spatula tips maintain full tip contact for relatively large lateral tip displacements. . . . . . . . . . . . . . . . . . . . . . . . 14

10 Experimental adhesion force vs. preload results compared with theoretical predictions. . . . . 